\section{Le programme d'éradication de la lucilie bouchère d'Afrique du Nord*}

\author{
E.P. Cunningham '
}

\author{
M. Abusowa ${ }^{2}$ \\ D.A. Lindquist ' \\ A.E. Sidahmed 1 \\ M. Vargas-Terán ${ }^{1}$
}

CUNNINGHAM (E.P.), ABUSOWA (M.), LINDQUIST (D.A.), SIDAHMED (A.E.), VARGAS-TERÁN (M.). Le programme d'éradication de la lucilic bouchc̀re d'Afrique du Nord. Revue Élev. Méd. vét. Pays trop., 1992, 45 (2) : 115-118

La lucilie bouchère (Cochliomyia hominivorax) est un parasite essentiel des animaux à sang chaud. La femelle peut pondre jusqu'à 300 oeufs dans n'importe quelle blessure, si petite soit-elle, et les larves qui en résultent ("vers") fouissent la chair vive des animaux. Beaucoup d'animaux infestés meurent, alors que le coût annuel de la lutte contre cette infestation chez les animaux domestiques est d'environ 10 dollars par tête. La lucilie bouchère est endémique en Amérique latine tropicale. En 1988, elle a été détectée pour la première fois en Libye où elle a pu être introduite par des moutons importés. En 1990, l'infestation s'était propagée sur une zone de $25000 \mathrm{~km}^{2}$, où vivent quelque 2 millions de têtes de bétail. Début 1991, la FAO a entrepris un programme d'éradication par la méthode de l'insecte stérile financé par la communauté internationale. Chaque semaine, 40 millions de pupes ont été expédiées par avion de l'usine de production située au Mexique pour être lâchées, une fois parvenues à maturité, au-dessus de la zone infestée. En quelque mois, l'infestation a été éradiquée. Alors qu'en 1990, 12000 animaux infestés avaient été détectés, six cas seulement ont été enregistrés l'année suivante. Le programme prévoyait l'expédition et le lâcher de 1,3 milliard d'insectes stériles, des inspections d'animaux (40 millions au total) et l'examen en laboratoire de 280000 mouches capturées. Le programme ayant coûté au total près de 75 millions de dollars E.-U., le rapport coût/bénéfice a été estimé à 1:50. Mots clés : Lucilie bouchère Cochliomyia hominivorax - Éradication - Lâcher d'insectes stériles Libye - Afrique du Nord.

\section{Introduction}

La lucilie bouchère (Cochliomyia hominivorax, Coquerel) est un diptère dont la larve est un parasite essentiel des animaux à sang chaud. Elle a été signalée pour la première fois il y a plus d'un siècle (COQUEREL, 1858) dans des échantillons prélevés dans les sinus infectés de bagnards à l'île du Diable en Guyane française (2).

A la température préférée $\left(20-30^{\circ} \mathrm{C}\right)$, le cycle biologique de la lucilie bouchère est d'environ 21 jours. La femelle, qui ne s'accouple qu'une fois, peut pondre ses oeufs en un ou plusieurs lots pouvant compter jusqu'à 300 oeufs

1. Organisation des Nations unies pour l'alimentation et l'agriculture (FAO), Viale delle Terme di Caracalla, 00100 Rome, Italie.

2. Directeur national du Programme, Programme de terrain du SECNA, Tripoli, Libye.

* Ce travail a été présenté au sixième congrès international sur la Reproduction des invertébrés, Dublin, Irlande, juillet 1992.

Reçu le 8.9.1992, accepté le 16.9.1992. chacun, sur le bord de toute blessure ou coupure d'un animal à sang chaud, y compris dans des blessures aussi petites que des morsures de tique, ainsi que dans les orifices naturels. Les larves se développent en 24 h et fouissent la chair vive, de sorte que la blessure s'approfondit et s'élargit et attire un plus grand nombre de mouches femelles qui, à leur tour, pondent d'autres oeufs. Si ces blessures ne sont pas soignées elles sont souvent fatales, notamment chez l'animal qui vient de naître, car l'insecte dépose habituellement ses oeufs dans l'ombilic.

La lucilie bouchère est originaire des régions tropicales du continent américain. Elle peut se reproduire tout au long de l'année à des latitudes situées entre $30^{\circ}$ Nord et $30^{\circ}$ Sud (approximativement de l'Argentine au Texas) et, selon les saisons, jusqu'à $1000 \mathrm{~km}$ au-delà de ces limites, au nord comme au sud. Elle ne s'est jamais établie en dehors du continent américain jusqu'en 1988.

Dans sa région endémique, la lucilie bouchère est l'insecte le plus nuisible au bétail domestique. Avant qu'elle ne soit éradiquée des États-Unis, les pertes étaient estimées à 300 millions de dollars par an pour le seul État du Texas. Dans ces régions, on luttait contre cette infestation en inspectant régulièrement le bétail, en soignant toutes les blessures et zones d'infection potentielles avec des insecticides, pour un coût annuel de quelque 10 dollars E.-U. par tête de bétail.

Après quelques années d'expérimentation des techniques de production en masse et de stérilisation par irradiation (1), les premiers essais sur le terrain de la technique de l'insecte stérile ont eu lieu sur Sanibel Island, au large de la Floride, en 1953. Ils ont été suivis d'une campagne d'éradication réussie sur l'île de Curaçao en 1954 . puis d'une autre campagne d'éradication en Floride de 1957 à 1959. Entre 1962 et 1966, les États du sud des Étas-Unis se sont débarrassés de la lucilie bouchère grâce à des campagnes successives utilisant la technique de l'insecte stérile. Toutefois, comme de nouvelles infestations se produisaient régulièrement à cause d'insectes provenant du Mexique, une nouvelle campagne a été organisée conjointement par le Mexique et les États-Unis entre 1976 et 1990 et a abouti à l'éradication complète de l'insecte du Mexique (4). Des campagnes identiques se poursuivent en Amérique centrale, dans le but d'éradiquer la lucilie bouchère de tous les pays situées au nord du Panama et de constituer une barrière permanente d'insectes stériles en travers de l'isthme du Panama.

\section{Infestation en Afrique du Nord}

Dans les premiers mois de 1988, des agriculteurs libyens ont signalé des myiases (blessures infestées de larves) inhabituelles qui ont été examinées par la Faculté vétérinaire de l'Université Al-Fateh de Tripoli. Quelques mois plus tard, des échantillons ont été envoyés au British Museum et la présence de la lucilie bouchère a été confirmée (3). L'inspection systématique des animaux et les contrôles zoosanitaires ont commencé au milieu de l'année 1989, tandis qu'une augmentation régulière de 


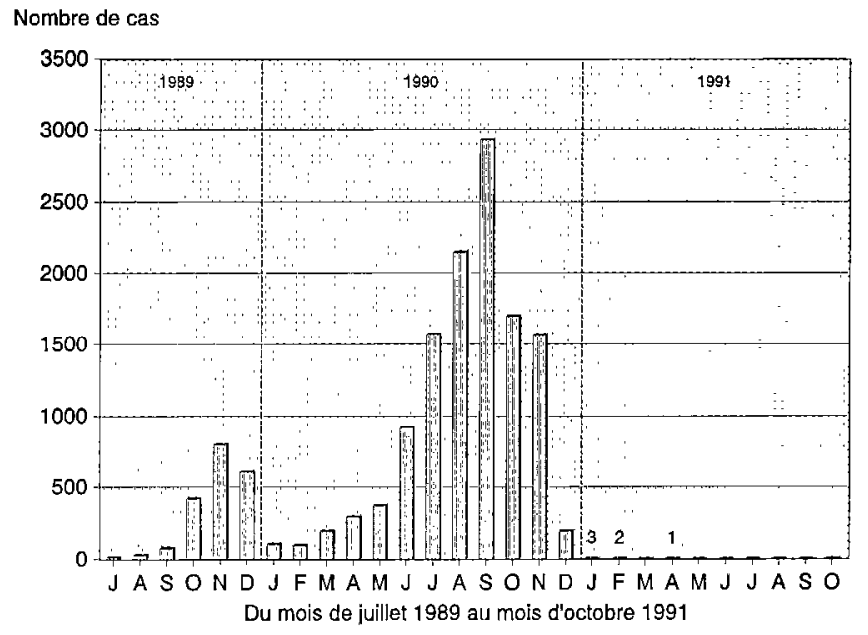

Figure 1 : Cas de myiase de la lucilie bouchère en Libye (juillet 1989octobre 1991).

l'incidence des animaux infestés était signalée dans une zone s'étendant sur $150 \mathrm{~km}$ le long de la côte méditerranéenne de chaque côté de Tripoli et jusqu'à $100 \mathrm{~km}$ à l'intérieur. Le nombre des animaux infestés a baissé pendant l'hiver, pour augmenter spectaculairement au printemps de 1990 et atteindre 3000 cas au mois de septembre (fig. 1).

Confronté à la perspective d'un combat indéfini contre ce nouveau ravageur, et conscient du danger qu'il représentait pour le continent africain et le sud de l'Europe, le Gouvernement libyen a demandé une assistance extérieure. Un certain nombre d'organisations internationales, ainsi que beaucoup de pays traditionnellement donateurs d'aide internationale, ont répondu. La FAO a fourni une assistance immédiate pour le traitement, la surveillance et le contrôle zoosanitaires et a aidé les sept pays ayant une frontière commune avec la Libye à renforcer leurs mesures de quarantaine et à mettre en place des systèmes de surveillance des animaux.

II est apparu très vite que ces mesures visant à limiter la propagation de la maladie ne pouvaient à elles seules éradiquer le ravageur, ni prévenir sa diffusion. Des plans pour une campagne d'éradication fondée sur la méthode de l'insecte stérile ont donc été établis et c'est sur cette base qu'un appel a été lancé à la communauté internationale. En se fondant sur l'expérience acquise en Amérique du Nord, le plan prévoyait une campagne d'éradication sur deux années. Plusieurs options ont été envisagées pour la fourniture de mouches stériles, y compris la construction d'un centre de production en Libye. Toutefois, la nécessité d'agir rapidement ainsi que les investissements importants que cela aurait représenté, ont fait préférer le transport par avion de mouches stériles provenant du seul centre de production existant aujourd'hui dans le monde situé à Tuxtla Guttierez, au sud du Mexique. Au moment où le programme a été lancé, le centre produisait 140 millions de mouches par semaine pour des campagnes d'éradication en Amérique centrale. Grâce à des investissements supplémentaires, sa capacité s'est accrue de 100 millions d'insectes par semaine et des contrats pour la fourniture suivie de mouches stériles nécessaire au programme pour l'Afrique du Nord ont été rédigés. Ces arrangements n'ont pas été faciles à conclure car l'usine appartient à la fois aux gouvernements mexicain et américain et ni l'un ni l'autre n'a de relations diplomatiques avec la Libye. Un décret spécial a dû être promulgué pour permettre à la Commission mixte Mexique-États-Unis pour l'éradication de la lucilie bouchère, de vendre des insectes au programme.

Le coût de ce programme d'une durée de deux ans était estimé à plus de 100 millions de dollars E.-U. Outre les quelque 25 millions de dollars E.-U. que le Gouvernement libyen demandait pour appuyer les opérations au sol, le coût de l'achat des insectes, de leur transport par avion et de leur lâcher au-dessus de la zone infestée, y compris celui des activités d'appui nécessaires, a été estimé à 91 millions de dollars E.-U. Lors d'une réunion de donateurs tenue à Rome en janvier 1990, des contributions ont été annoncées pour un montant total de 31 millions de dollars E.-U. L'organisation de la campagne d'éradication a été confiée à la FAO.

Les inventeurs de la technique de l'insecte stérile ont calculé qu'avec une proportion de dix mâles stériles pour un mâle fertile dans la population sauvage, il faudrait environ dix cycles biologiques pour que l'éradication soit complète (5). On avait donc tout intérêt soit à tenter de supprimer un nombre maximum d'insectes sauvages avant de lancer la compagne, soit à tirer parti des diminutions saisonnières de la population d'insectes. La campagne a donc été planifiée de façon à démarrer au milieu de l'hiver 1990/91, époque à laquelle, d'après l'expérience acquise, la population serait à son niveau le plus bas. Huit livraisons hebdomadaires ont donc été faites à titre d'essai à partir du 14 décembre, les insectes étant dispersés sur un quart environ de la zone infestée. Ces essais visaient à vérifier la viabilité des insectes après un transport exceptionnellement long et à tester la logistique de l'opération sous tous ses aspects.

\section{Qualité des insectes}

L'efficacité des insectes stériles a pour ultime critère l'élimination de la population sauvage. Pratiquement, on la mesure à la proportion de masses d'oeufs stériles prélevés sur les animaux sentinelles. Toutefois, ce procédé n'est concluant que si un nombre raisonnable de masses d'oeufs peut être prélevé, ce qui suppose soit une forte densité de population, soit un grand nombre d'animaux sentinelles. On peut mesurer également le degré de compatibilité sexuelle entre les souches produites par l'usine et la population sauvage, ainsi que le pourcentage de pupes donnant des insectes adultes et la capacité de ces derniers de voler et de survivre.

La compatibilité sexuelle des insectes a été évaluée grâce à des tests d'accouplement réciproque entre la souche actuellement produite par l'usine du Mexique et la population sauvage (6). Les tests de compatibilité ont été 
entièrement positifs. En outre, la similarité génétique entre la souche libyenne d'une part, et celle produite par l'usine et d'autres souches mexicaines et des Caraïbes d'autre part, a été mesurée en comparant des fragments d'ADN mitochondrial produits par 15 enzymes de restriction (6). L'analyse du site de restriction a permis de conclure que la souche libyenne n'est originaire ni de la Jamaïque, ni du Mexique, ni de l'Amérique centrale.

Cinquante quatre expéditions de pupes ont été organisées entre le Mexique et Tripoli. Pour chaque livraison allant de 3,5 millions à 40 millions de pupes, et pour chaque lot dans le cas des expéditions les plus importantes, des tests de contrôle de qualité ont été effectués sur des échantillons prélevés avant le départ du Mexique et après l'arrivée à Tripoli. La figure 2 permet de comparer les résultats obtenus mois par mois, au départ et à l'arrivée. on constate que les taux d'émergence d'insectes adultes et les taux d'agilité ne diffèrent pratiquement pas. Quant aux tests de longévité, ils indiquent des valeurs supérieures à l'arrivée à Tripoli pour 8 des 11 mois considérés. Ces résultats confirment que la qualité des insectes n'est pratiquement pas affectée par le transport et le stockage.

\section{La campagne d'éradication}

Les premières expéditions ont été faites par un itinéraire compliqué, qui impliquait un transport par camion de $24 \mathrm{~h}$, suivi d'un vol de $8 \mathrm{~h}$ dans la soute d'un avion de ligne, puis d'un vol affrété de $3 \mathrm{~h}$ entre Francfort et Tripoli. Lorsqu'il a fallu importer des quantités plus importantes, on a préféré, pour des raisons économiques complexes, organiser un transport par vols directs dans de gros appareils affrétés. Une fois arrivés à destination, il était essentiel que les insectes soient immédiatement disponibles et en bonne condition de façon que l'ensemble de la zone infestée puisse être traité tous les 3 ou 4 jours. Un travail de planification très minutieux a été nécessaire pour répondre à toutes ces conditions. II y aurait un départ par semaine, chaque avion transportant 40 millions de mouches préemballées dans des boîtes prêtes à être lâchées et palettisées. Chaque livraison comprenait des lots de pupes parvenues à cinq stades différents de maturation. A l'arrivée à Tripoli elles étaient chargées dans des remorques réfrigérées et transportées vers les zones infestées par étapes, en variant les températures, de façon à assurer un approvisionnement continu en insectes d'excellente qualité et prêts à être lâchés pendant les 4 jours suivants.

La zone infestée en Libye couvrait $25000 \mathrm{~km}^{2}$. Avec la zone tampon, qui englobait notamment $5000 \mathrm{~km}^{2}$ de territoire tunisien, la superficie totale à traiter était de $40000 \mathrm{~km}^{2}$, soit un territoire à peine inférieur à celui des Pays-Bas.
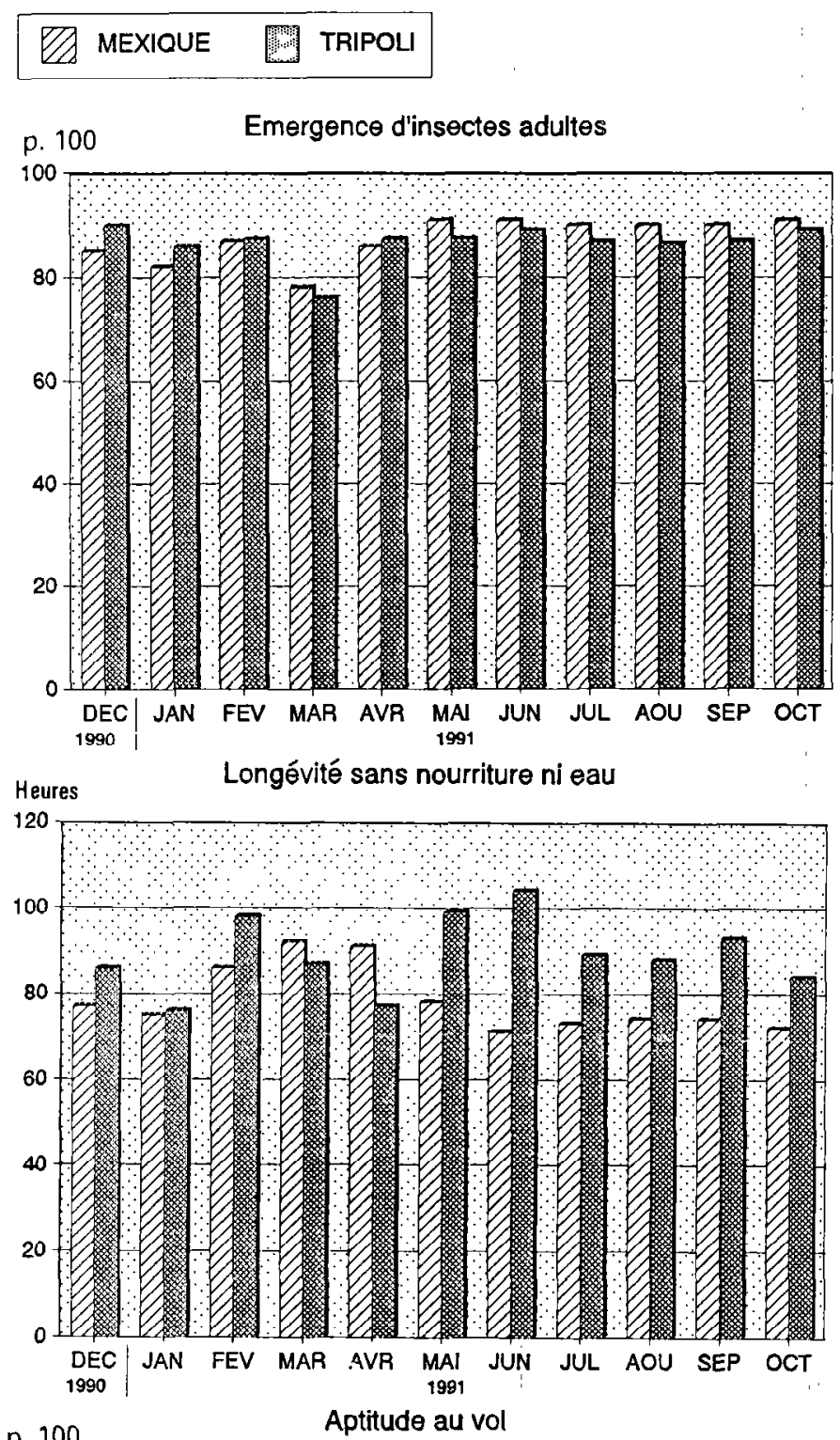

p. 100

Aptitude au vol

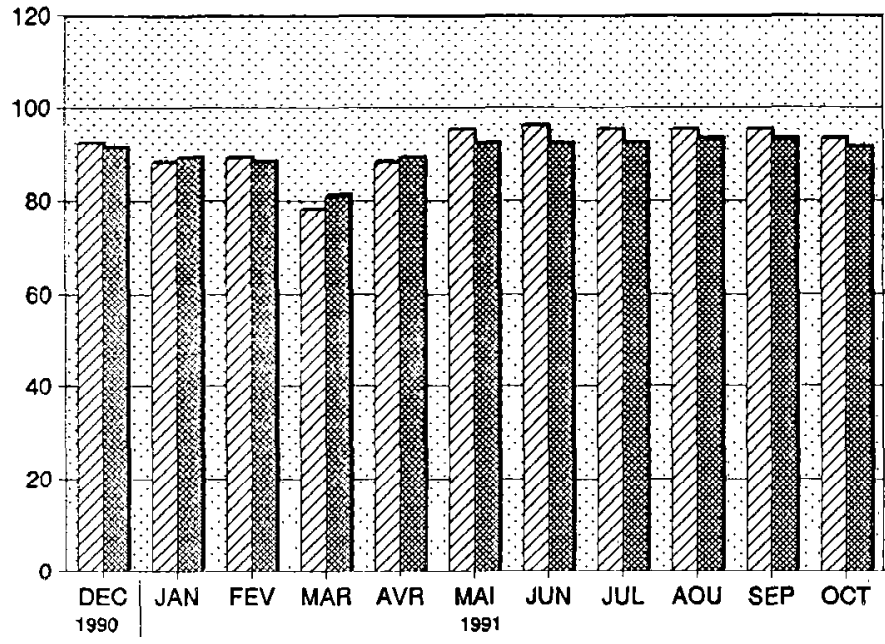


Cinq appareils spécialement équipés lâchaient les mouches sur des couloirs aériens espacés l'un de l'autre de $4 \mathrm{~km}$, de façon à obtenir une concentration moyenne de 1000 insectes par $\mathrm{km}^{2}$, bien que les écarts soient assez grands d'une zone à l'autre et d'un secteur à l'autre, et varient selon les estimations de la densité de la population sauvage.

Le traitement de la zone infestée a commencé début février et semble avoir été efficace dès le début. L'expansion de la population, qui se produit normalement au printemps, n'a tout simplement pas eu lieu. Alors que 12000 cas avaient été détectés l'année précédente, on n'en a observé que six en 1991, le dernier s'étant produit en avril. La pose massive de pièges à mouches (82 pièges permettant de capturer quelque 20000 mouches par mois) n'a révélé que 23 cas de lucilie bouchère fertile, là encore pendant les quatre premiers mois de l'année uniquement.

Lorsque l'éradication est en bonne voie, la population sauvage diminue rapidement et sa densité tombe à un niveau très bas, ce qui rend extrêmement difficile la détection de tout résidu de la population. On continue par conséquent à lâcher des insectes stériles pendant les 9 cycles biologiques suivant la date à laquelle a été repéré le dernier survivant. C'est la méthode qui a été adoptée pour la présente campagne, avec une distribution hebdomadaire de 40 millions d'insectes stériles jusqu'au 15 octobre 1991, après quoi la campagne a été arrêtée.

Depuis lors, la surveillance intense du bétail, la pose de pièges à insectes et les contrôles zoosanitaires se poursuivent. Chaque mois, tout le cheptel de la région ( 2 millions de têtes) est inspecté au moins une fois, quelque 20000 mouches sont capturées et 50000 animaux font l'objet de contrôles sanitaires. Aucune trace de lucilie bouchère vivante n'ayant été détectée, la campagne d'éradication a été déclarée close le 22 juin 1992.

\section{Conclusion}

Une évaluation indépendante des coûts et bénéfices de ce programme a été entreprise. Si l'on tient compte de toutes les dépenses, supportées par toutes les parties et par les pays voisins, tant en espèces qu'en nature, le coût total de la campagne est estimé à quelque 75 millions de dollars $\mathrm{E}$.$U$. Les bénéfices ont été calculés uniquement sur la base des économies réalisées en matière d'inspection et de traitement de routine des animaux, de la réduction de la mortalité et des meilleures performances des animaux en l'absence de lucilie bouchère. Les bénéfices nets actualisés ont ensuite été calculés pour la zone infestée, pour l'ensemble de la Libye et pour l'ensemble de l'Afrique du Nord. Le rapport bénéfice/coût a été estimé pour chacune de ces trois zones à 1,5:1, 6:1 et 50:1, respectivement.

CUNNINGHAM (E.P.), ABUSOWA (M.), LINDQUIST (D.A.), SIDAHMED (A.E.), VARGAS-TERÁN (M.). The New World Screwworm eradication programme in North Africa. Revue Élev. Méd. v'ét. Pays trop., 1992, 45 (2) :115-118

The New World Screwworm (NWS, Cochliomyia hominivorax) is an obligate parasite of warm-blooded animals. The female lays up to 300 eggs in any break in the skin, and the resulting larvae (screwworms) burrow into surrounding living flesh. Infested animals frequently die, while the annual cost of controlling the pest in domestic animals is about US\$10 per head.
NWS is endemic in tropical Latin America. In 1988, it was detected in Libya, presumable introduced with imported sheep. By 1990, the infestation had spread to an area of $25,000 \mathrm{~km}^{2}$ containing somo 2 million livestock. In early 1991, an internationally funded eradication programme was undertaken by FAO, using sterile insects. Each week, 40 million pupae were flown from a production plant in Mexico, and the emerged adults were distributed by over the infested area. Within a few months, the infestation has been eradicated. Whereas 12,000 infested animals were found in 1990 , only 6 were detected in 1991 . The programme involved the shipping and distribution of 1.3 billion sterile insects, animal inspections totalling 40 million and laboratory examination of 280,000 trapped flies. While the programme cost close to US\$75 million, a benifit/cost ration of 50:1 has been estimated; Key words : New World Screwworm - Cochliomyia hominivorax - Eradication - Sterile insect release - Libya - North Africa.

\section{Bibliographie}

1. BUSHLAND (R.C.), HOPKINS (D.E.). Experiments with screwworm flies sterilized by X-rays. J. econ. Ent., 1951, $44: 725-731$.

2. COQUEREL (Ch.). Des larves de diptères développées dans les sinus frontaux et les fosses nasales de l'homme, à Cayenne. Paris, Archives Générales de Médecine, 1858: 513-528.

3. EL-AZAZY (O.M.E.). Wound myiasis caused by Cochliomyia hominivorax in Libya. Vet. Rec., 1989. 124 (4) : 103.

4. GRAHAM (O.H.) Ed. Symposium on Eradication of the Screwworm from the United States and Mexico. Vol. 62. Maryland, Entomological Society of America, 1985: 1-66.

5. KNIPLING (E.P.). Possibilities of insect control or eradication through the use of sexually sterile males. J. Econ. Ent. $1955,48: 459-462$.

6. TAYLOR (D.B.), HAMMACK (L.), ROEHRDANZ (L.). Reproductive compatibility and mitnchondrial DNA restriction site analysis of New World Screwworm, Cochliomyia hominivorax, from North Africa and Central America. Med. Vet. Ent., 1991, 5 : 145-151.

\section{Communication}

\section{Une épidémie de kératoconjonctivite granuleuse ovine, d'origine chlamydienne, en Côte-d'Ivoire}

\author{
P. Formenty 1
}

\section{J. Domenech ${ }^{2}$}

FORMENTY (P.), DOMENECH (J.). Une épidémie de kératoconjonctivite granuleuse ovine, d'origine chlamydienne, en Côte-d'Ivoire. Revue Élev. Méd. vét. Pays trop., 1992,45 (2) : 118-120

Au cours de la saison sèche 1990-1991, une épidémie de kératoconjonctivite granuleuse ovine a sévi en Côte-d'Ivoire. L'étiologie chlamydienne de l'affection a été démontrée. Tous les troupeaux ont été atteints avec une morbidité variant de 30 à $70 \mathrm{p}$. 100 . Les lésions de kératite ont été observées sur 5 à 15 p. 100 des malades. Le traitement à l'Auréomycine (NDSpecia, pommade ophtalmique) s'est avéré constamment efficace. Mots clés : Ovin - Kératoconjonctivite - Chlamydia - Antibiotique Auréomycine - Côte-d'Ivoire.

1. Laboratoire central de pathologie animale, BP 206, Bingerville, Côte-d'lvoire.

2. OUA-IBAR, POB 30786, Nairobi, Kenya.

Reçu le 7.4.1992, accepté le 20.4.1992. 